\title{
INVENTORY DECISION IN VUCA WORLD USING ECONOMIC LOGIC QUANTITY
}

\author{
Lidia Vesa ${ }^{1}$, Marcel loan Boloș ${ }^{2}$, Claudia Diana Sabău-Popa ${ }^{2}$ \\ Doctoral School of Economic Sciences, Faculty of Economic Sciences, University of Oradea, \\ Romania, \\ University of Oradea, Faculty of Economic Sciences, Oradea, Romania \\ lidiavesa@gmail.com \\ marcelbolos@yahoo.com \\ dianasabaupopa@yahoo.ro
}

\begin{abstract}
If ever the concept "VUCA" (Volatility, Uncertainty, Complexity, and Ambiguity) seemed appropriate to use, it is now. National and global companies experience the highest level of instability due to the Covid-19 pandemic, which is the classic example of a highly volatile, uncertain, complex, and ambiguous world. In this world, decision-makers have to face more challenges appealing to the VUCA Prime leadership approach: vision against volatility, understanding against uncertainty, clarity against complexity, and agility against ambiguity. Some of the ways through which managers can overcome the VUCA characteristics include: providing a shared vision as a criterion for all decisions to be made, identifying the reason for the decision problems and sharing the idea with the followers, going through the entire decision process, following steps in proper order, and developing quick solutions. In an inventory decision taken in a VUCA context, the above ways are possible if using fuzzy inventory methods dealing with volatility, uncertainty, complexity, and ambiguity. This paper aims to adapt a traditional inventory method, Economic Production Quantity (EPQ), to the challenges of the VUCA world, through the fuzzy logic system (FLS). To achieve the best solution for the decision problem in the shortest time possible, the managers can employ a conversion by using the computing platform MATLAB. There are some advantages of this conversion for these two methods, EPQ and FLS. Firstly, the transformation of EPQ in ELQ (Economic Logic Quantity) allows managers to formulate the decision problem, even if they cannot identify and measure precisely the EPQ parameters. Secondly, using FLS to solve ELQ provides the possibility to simulate more alternatives and generate the solution in the shortest amount of time. Thirdly, it allows the decision-makers to evaluate the impact of the solution provided by each simulation on the company's performance. Using these methods has the following primary limit: the problem formulation step depends on the managers' understanding ability and managing a large volume of information. Therefore, there may be a risk of obtaining a relevant solution for a decision problem if the decision-makers do not understand the cause of the problem or do not know how to organize and manage a large volume of information. This limit could be overcome by using AHP (Analytic Hierarchy Process), but this is the topic of further research.
\end{abstract}

Keywords: fuzzy logic system, economic production quantity, demand, cost, fuzzy inference, fuzzification, defuzzification.

JEL Classification: C53; C87; D24; $M 11$.

\section{Introduction}

The Economic Production Quantity is "a simple mathematical model to deal with inventory management issues in a production inventory system" (Viji and Karthikeyan, 2018). It is used to identify the optimal quantity to produce something in a production run. Also, it is used to measure the length of the production run, the reorder level, the average inventory, and the maximum inventory. This model has the following assumptions: the input variables are 
known and constant, the demand is fully satisfied, and therefore, the model does not allow shortages. Also, in the EPQ model, a single product is considered and is delivered at a single time. These assumptions are relevant in an abstract world, but in the real world, and more specifically in a VUCA world, the EPQ model with these assumptions is less applicable. That is why many researchers have enhanced and expanded the EPQ model to increase their application by introducing the quality concept, possible backorders and shortages, imperfect items at a discounted price, the multiple-item possibility, and, more recently, fuzzy numbers. Using fuzzy numbers in the EPQ model firstly means to transform the crisp variables of traditional EPQ into fuzzy variables according to some rules defined by the decision problem. Secondly, it means to transform the fuzzy results of EPQ to crisp results. This transformation is possible through the Fuzzy Logic System (FLS), which is "a special rule-based system that uses fuzzy logic and contains fuzzy rules in its knowledge base. It also derives conclusions from the user inputs and the fuzzy inference rules to map numeric data into variable linguistic terms and to make fuzzy reasoning work" (Kaur and Kaur, 2009).

In this paper, the combination between traditional EPQ and SLF provides a new model of Fuzzy EPQ (FEPQ) that is called: Economic Logic Quantity (ELQ). This ELQ is a more useful method than EPQ when the inventory decision is taken in a VUCA world due to the following characteristics:

- The variables can be easily adapted to changes in the decision process through fuzzy numbers that are formulated on a platform called MATLAB. Using this platform, the decisionmakers can simulate an infinite number of changes. This results in overcoming the volatility of VUCA.

- The model deals with vague information through fuzzy numbers. In an uncertain decisionmaking environment, managers cannot identify or estimate the correct value of each variable due to the lack of information. However, through fuzzy numbers, they can formulate decision variables in ranges or triangular and trapezoidal numbers, not only in real numbers. Also, through fuzzy numbers, the ambiguity of the decision process can be reduced.

- The model provides information about the risk that decision-makers should take when the input and output have certain values through the membership function of fuzzy numbers. This characteristic is very important in prediction, especially when the future is uncertain, because the managers will know if their prediction will be $100 \%$ achievable or not. Through this model, they will evaluate the impact of each level of feasibility on the future performance.

\section{Short literature review}

There are many articles and books that have used fuzzy logic or fuzzy numbers in the EPQ model. Lee and Yao (1998) fuzzified the production quantity in fuzzy triangular numbers for the first time. Lin and Yao (2000) used a trapezoidal fuzzy number for production quantity to solve the EPQ model. Hsieh (2002) analysed two cases of the FEPQ model (Fuzzy Economic Production Quantity), compared both cases to crisp EPQ and concluded that crisp $E P Q$ is equivalent to one of the two cases. Chen and Chang (2008) developed a FEPQ model for imperfect production. Roy et al. (2009) analysed an EPQ model with imperfect items that could be remanufactured at the quality that satisfies the customers. Paul et al. (2014) formulated a model with imperfect quality. There are many other extensions of the fuzzified EPQ model: considering an investment for reducing holding cost and setup cost (Islam and Roy, 2006), multi-item production (Mandal and Roy, 2006), shifting in production (Zhaet al. al, 2009), formulating a model with intuitionistic numbers (Chakrabortty, 2013), considering multi-period production (De and Sana, 2014) and partial back-ordering and disruption (De and Mahata, 2019).

Despite these multiple extensions, only a single article proposes a FLS to obtain an optimal solution for the production system. Miret et. al (2018) formulated a FLS with three inputs: demand, cost, and lead time represented by fuzzy triangular numbers and trapezoidal fuzzy 
numbers. The authors do not use all of the traditional EPQ inputs and thus could not compare the results of traditional EPQ and fuzzy EPQ.

This paper proposes a new model that uses the traditional formula of EPQ to obtain the variable values that fully belong to a fuzzy set or fuzzy number, to develop a FLS using EPQ inputs (demand, setup cost, holding cost, and production rate), and to compare the FLS output to traditional EPQ output. These steps leads to a new concept ELQ, which is the crisp solution for a Fuzzy EPQ, obtained using FLS.

\section{Inventory decision using ELQ}

\subsection{Modelling ELQ through FLS and EPQ formula}

ELQ is developed by using traditional EPQ inputs and formula in the first step of FLS formulation. The formula of EPQ is:

$$
E P Q=\sqrt{\frac{2 K D}{h\left(1-\frac{D}{P}\right)}} \quad, \begin{aligned}
& \text { where: } \mathrm{K}-\text { setup cost } \\
& \mathrm{D} \text {-demand } \\
& \mathrm{h}-\text { holding cost } \\
& \mathrm{P} \text { - production rate }
\end{aligned}
$$

Considering this formula, the inputs variables of FLS are: setup cost, demand, holding cost, production rate and the output variable is: ELQ. These variables should be formulated in fuzzy numbers, in the first step of FLS, evaluated by a rule base, in the second step, and transformed in crisp values, in the third step. The three steps of Fuzzy Logic System structure are presented below.

\section{A. The fuzzification step}

The fuzzification step consists in converting each crisp input in fuzzy input that has a universe of discourse, a membership function and an associated linguistic term.

The first input variable is setup cost which is described by three linguistic terms: low, reasonable and high and is defined by Definition 1 .

Definition 1: Let the discourse universe consists of the setup costs set $\mathrm{K}=\left[\mathrm{K}_{\mathrm{L}}, \mathrm{K}_{\mathrm{H}}\right]$ and the set of rules $F(0,1)$ valid for all fuzzy subsets described by the linguistic terms. The fuzzy numbers: $K_{I} \in K$, described by linguistic term: "low", $K_{r} \in K$, described by linguistic term "reasonable" and $\mathrm{K}_{\mathrm{h}} \in \mathrm{K}$, described by linguistic term: "high" are triangular fuzzy numbers of the form $k_{l}=\left(\begin{array}{llll}k_{l} & k_{l_{b}} & k_{c}\end{array}\right), k_{r}=\left(\begin{array}{lllll}k_{a} & k_{r_{b}} & k_{r_{c}}\end{array}\right)$ and $k_{h}=\left(\begin{array}{llll}k_{h_{a}} & k_{h_{b}} & k_{h_{c}}\end{array}\right)$, if their membership functions are defined by the following relation:

$$
\mu_{\mathrm{kl}, r, \mathrm{~h}}(\mathrm{X})=\left\{\begin{array}{cc}
O, & k_{l, r, h_{x}} \leq k_{l, r, h_{a}} ; k_{l, r, h_{x}} \geq k_{l, r, h_{c}} \\
\frac{k_{l, r, h_{x}}-k_{l, r, h_{a}},}{k_{l, r, h_{b}}-k_{l, r, h_{a}}} & k_{l, r, h_{a}} \leq k_{l, r, h_{x}} \leq k_{l, r, h_{b}} \\
\frac{k_{l, r, h_{c}}-k_{l, r, h_{x}}}{k_{l, r, h_{c}}-k_{l, r, h_{b}}} & k_{l, r, h_{b}} \leq k_{l, r, h_{x}} \leq k_{l, r, h_{c}} \\
1, & k_{l, r, h_{x}}=k_{l, r, h_{b}}
\end{array}\right.
$$

The graphical representation for this fuzzy numbers is illustrated in the following figure:

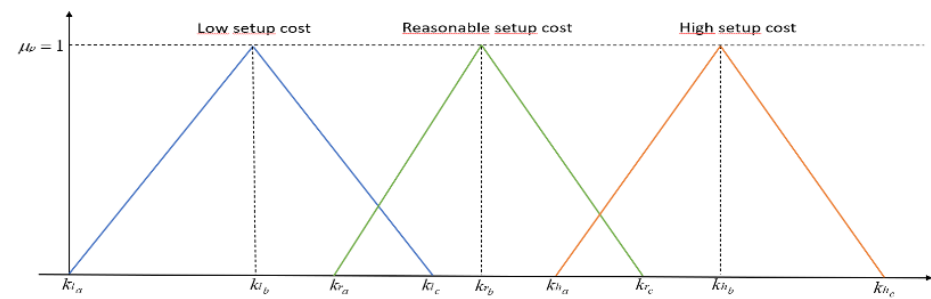

Figure Nr. 1: Setup costs and membership functions of the three setup costs 
The second input, demand is described, also, by three linguistic terms: low, medium and high and is defined by Definition 2 .

Definition 2: Let the discourse universe consists of the setup costs set $D=\left[D_{\llcorner}, D_{H}\right]$ and the set of rules $F(0,1)$ valid for all fuzzy subsets described by the linguistic terms. The fuzzy numbers: $D_{1} \in D$, described by linguistic term: "low", $D_{m} \in D$, described by linguistic term "medium" and $D_{h} \in D$, described by linguistic term: "high" are triangular fuzzy numbers of the form $\quad d_{l}=\left(\begin{array}{llll}d_{l_{a}} & d_{l_{b}} & d_{l_{c}}\end{array}\right), d_{m}=\left(\begin{array}{llll}d_{m_{a}} & d_{m_{b}} & d_{m_{c}}\end{array}\right)$ and $d_{h}=\left(\begin{array}{lll}d_{h_{a}} & d_{h_{b}} & d_{h_{c}}\end{array}\right)$, if their membership functions are defined by the following relation:

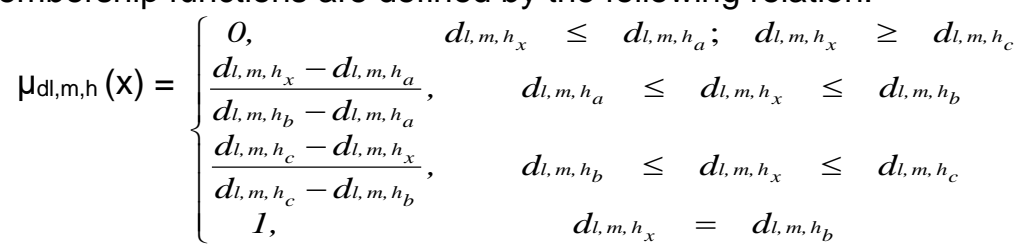

The graphical representation for this fuzzy numbers is illustrated in the following figure:

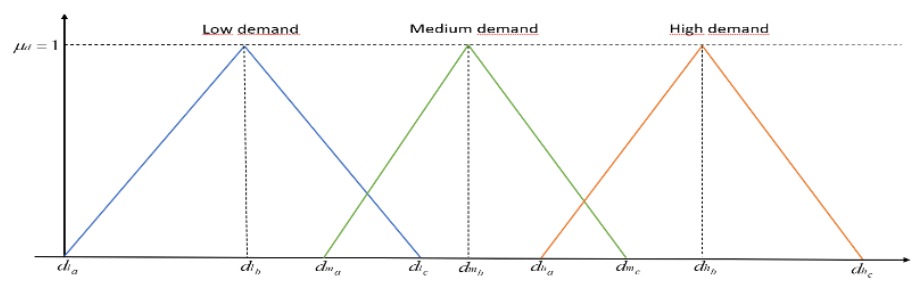

Figure Nr. 2: Demand and membership functions of the three categories of demand

As, setup cost, the holding cost, the third input variable, is described by the same linguistic terms: low reasonable and high and is defined by Definition 3.

Definition 3: Let the discourse universe consists of the setup costs set $\mathrm{H}=\left[\mathrm{H}_{\mathrm{L}}, \mathrm{H}_{H}\right]$ and the set of rules $F(0,1)$ valid for all fuzzy subsets described by the linguistic terms. The fuzzy numbers: $H_{l} \in H$, described by linguistic term: "low", $H_{r} \in H$, described by linguistic term "reasonable" and $\mathrm{H}_{\mathrm{h}} \in \mathrm{H}$, described by linguistic term: "high" are triangular fuzzy numbers of the form $h_{l}=\left(\begin{array}{llll}h_{l} & h_{l_{b}} & h_{l_{c}}\end{array}\right), h_{r}=\left(\begin{array}{llll}h_{a} & h_{r_{b}} & h_{r_{c}}\end{array}\right)$ and $h_{h}=\left(\begin{array}{lll}h_{h} & h_{h_{b}} & h_{h_{c}}\end{array}\right)$, if their membership functions are defined by the following relation:

$$
\mu_{\mathrm{hl}, \mathrm{r}, \mathrm{h}}(\mathrm{X})=\left\{\begin{array}{cc}
O, & h_{l, r, h_{x}} \leq h_{l, r, h_{a}} ; h_{l, r, h_{x}} \geq h_{l, r, h_{c}} \\
\frac{h_{l, r, h_{x}}-h_{l, r, h_{a}},}{h_{l, r, h_{b}}-h_{l, r, h_{a}}} & h_{l, r, h_{a}} \leq h_{l, r, h_{x}} \leq h_{l, r, h_{b}} \\
\frac{h_{l, r, h_{c}}-h_{l, r, h_{x}},}{h_{l, r, h_{c}}-h_{l, r, h_{b}}} & h_{l, r, h_{b}} \leq h_{l, r, h_{x}} \leq h_{l, r, h_{c}} \\
1, & h_{l, r, h_{x}}=h_{l, r, h_{b}}
\end{array}\right.
$$

The graphical representation of the fuzzy holding cost is illustrated in Figure Nr. 4.

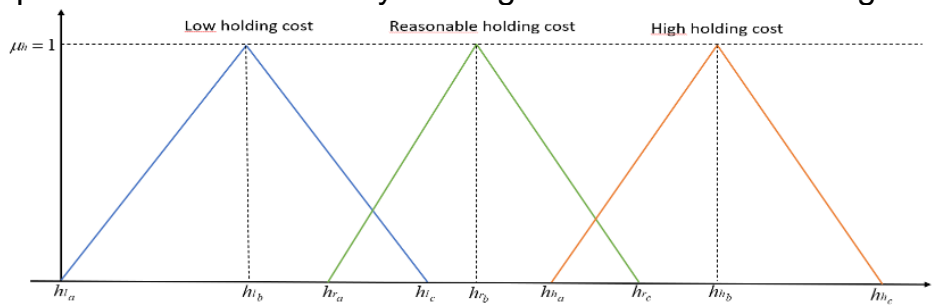

Figure Nr. 3: Holding cost and membership functions of the three holding costs 
The last input variable is production rate and is described by two linguistic terms: low and high, because the medium production rate is a crisp number.

Definition 4: Let the discourse universe consists of the setup costs set $\mathrm{P}=\left[\mathrm{P}_{L}, \mathrm{P}_{H}\right]$ and the set of rules $F(0,1)$ valid for all fuzzy subsets described by the linguistic terms. The fuzzy numbers: $P_{l} \in P$, described by linguistic term: "low" and $P_{h} \in P$, described by linguistic term: "high" are triangular fuzzy numbers of the form $p_{l}=\left(\begin{array}{llll}p_{l} & p_{l_{b}} & p_{l_{c}}\end{array}\right)$ and $p_{h}=\left(\begin{array}{lll}p_{h_{a}} & p_{h_{b}} & p_{h_{c}}\end{array}\right)$, if their membership functions are defined by the following relation:

$$
\mu_{\mathrm{pl}, \mathrm{h}}(\mathrm{X})=\left\{\begin{array}{lr}
O, & p_{l, h_{x}} \leq p_{l, h_{a}} ; p_{l, h_{x}} \geq p_{l, h_{c}} \\
\frac{p_{l, h_{x}}-p_{l, h_{a}}}{p_{l, h_{b}}-p_{l, h_{a}}}, & p_{l, h_{a}} \leq p_{l, h_{x}} \leq p_{l, h_{b}} \\
\frac{p_{l, h_{c}}-p_{l, h_{x}}}{p_{l, h_{c}}-p_{l, h_{b}}} & p_{l, h_{b}} \leq p_{l, h_{x}} \leq p_{l, h_{c}} \\
l, & p_{l, h_{x}}=p_{l, h_{b}}
\end{array}\right.
$$

The graphical representation of the fuzzy production rate is illustrated in the following figure:

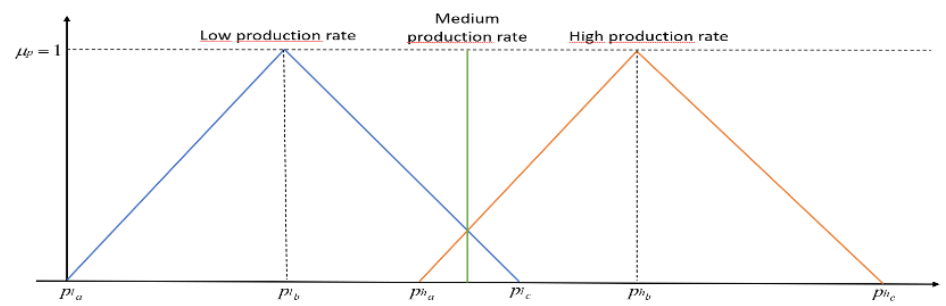

Figure Nr. 4: Production rate and membership functions

The output variable, ELQ (Economic Logic Quantity), is described through three linguistic variables: profitable (ELQ), zero profit (ELQ) and unprofitable (ELQ). These linguistic variables are used to provide information about the impact of quantity level on the company performance.

Definition 5: Let the discourse universe consists of the economic production quantity set $E L Q=\left[E L Q_{u}, E L Q_{p}\right]$ and the set of rules $F(0,1)$ valid for all fuzzy subsets described by the linguistic terms. The fuzzy numbers: $E L Q_{u} \in E L Q$, described by linguistic term: "unprofitable", ELQ $\in$ ELQ, described by linguistic term "zero profit" and ELQp $\in$ ELQ, described by linguistic term: "profitable" are triangular fuzzy numbers of the form $e l q_{u}=\left(\begin{array}{lll}e l q_{u_{a}} & e l q_{u_{b}} & e l q_{u_{c}}\end{array}\right), \quad \quad e l q_{0}=\left(\begin{array}{lll}e l q_{0_{a}} & e l q_{0_{b}} & e l q_{0_{c}}\end{array}\right) \quad$ and $e l q_{p}=\left(\begin{array}{lll}e l q_{p_{a}} & e l q_{p_{b}} & e l q_{p_{c}}\end{array}\right)$, if their membership functions are defined by the following relation:

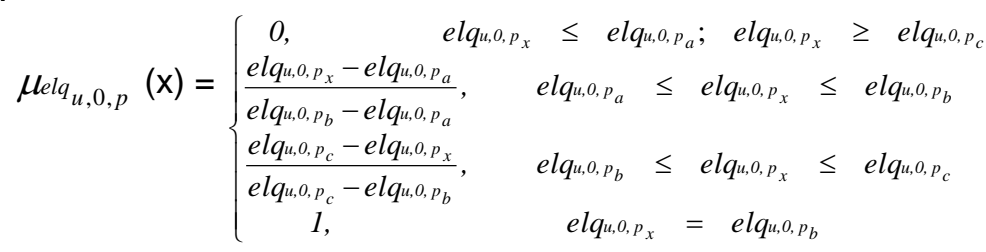

The graphical representation for the output value is illustrated in the following figure: 


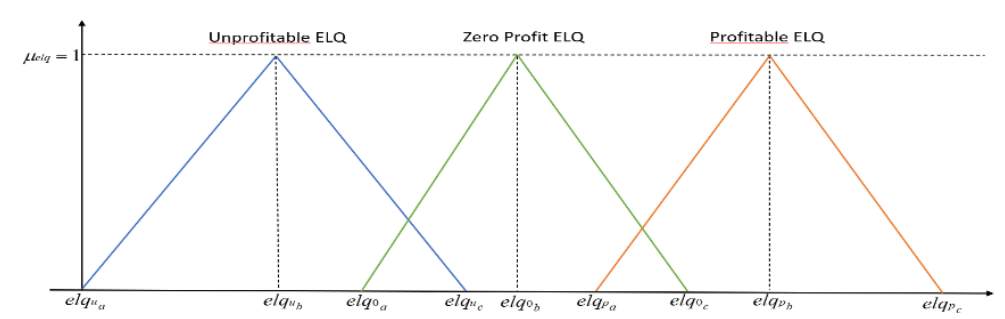

Figure Nr. 5: ELQ and membership functions

The values of the output ELQ for which $\mu_{e l q_{u, 0, p}}=1$, were determined by the ELQ formula as follows:

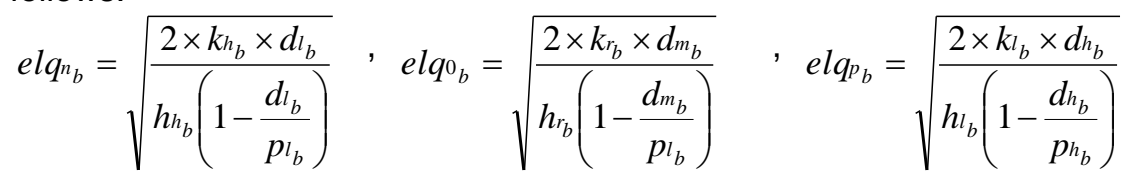

\section{B. The inference step}

After the formulation and fuzzification of the input and output variables, the FLS should have a rule base in order to provide a solution that consider all these variables. This rule base is constructed by the decision makers using" if-then" rules. For the above defined variables, the numbers of the rules in rule base is 54 (Appendix $A$ ).

The inference step consists in: evaluating each rules through union and intersection operation depending on the operator used in each rule between variables (or, and), combining the results of each rule and obtaining a graph for each rule. Through this step, the decision makers can evaluate the impact of each rule in the final solution.

There are two types of inferences: MAMDANI and SUGENO. In this paper, the MAMDANI inference is used.

\section{The deffuzification step}

This step has the following sub-steps:

- union of each rule graph;

- transformation of the solution, provided by the union of all rule graphs, from a fuzzy number to a crisp number. This is possible through defuzzification methods: centroid methods, weighted average method, min-max membership method and center of sums. Centroid method is the most widely used defuzzification method, especially when the FLS is solved through MATLAB platform, although the weighted average method is used. The formula of centroid method is:

$$
x^{*}=\frac{\int \mu_{A}(x) \cdot x d x}{\int \mu_{A}(x) d x}
$$

where: $\mu A(x)$ - membership degree for $x$ variable in each rule inference;

$x$ - variable value in each rule inference.

In this last step, the solution of the FLS is provided and it is a crisp value that belongs to discourse universe of the output variable. Therefore, the FLS solution is a crisp ELQ that is different from crisp EPQ. In order to compare EPQ and ELQ, it is necessary to test the traditional and fuzzy method. 


\subsection{Testing traditional EPQ and fuzzy ELQ method in an inventory decision}

\section{A. Testing traditional EPQ method}

Considering a manufacturing company, the input variables of the traditional EPQ are evaluated and ranked by the historical evolution of setup cost, demand, holding cost and production rate in the following table:

\begin{tabular}{|c|c|c|c|c|}
\hline & Setup Cost & Demand & Holding Cost & Production rate \\
\hline Low & $\$ 100$ & 400 qty. & $\$ 7$ & 700 qty. \\
\hline Medium/Reasonable & $\$ 130$ & 600 qty. & $\$ 11$ & 800 qty. \\
\hline High & $\$ 180$ & 900 qty. & $\$ 16$ & 1.100 qty. \\
\hline
\end{tabular}

These variable values were selected and ranked by the frequency of these values in some specific periods of time: with profitable production, with zero-profit production and with unprofitable production. For example, in a crisis period, with unprofitable production, the most frequent value for setup cost was $\$ 100$, thus, the lowest setup cost should be around $\$ 100$. The highest setup cost, due to the frequency of occurrence in the past, could be around $\$ 180$. In crisp EPQ, decision makers should accurately evaluate and rank the inputs, in order to obtain a valid solution. In fuzzy ELQ, decision makers can overcome the risk of errors in ranking, through fuzzy numbers.

According to the data from the Table 1, the managers asses three levels of EPQ:

- low EPQ

- medium EPQ

$$
E P Q_{1}=\sqrt{\frac{2 K_{l} D_{l}}{h_{l}\left(1-\frac{D_{l}}{P_{l}}\right)}}=\sqrt{\frac{2 \times 100 \times 400}{7 \times\left(1-\frac{400}{700}\right)}}=164 \text { qty. / production cycle }
$$

$$
E P Q_{m}=\sqrt{\frac{2 K_{r} D_{m}}{h_{r}\left(1-\frac{D_{m}}{P_{m}}\right)}}=\sqrt{\frac{2 \times 130 \times 600}{11 \times\left(1-\frac{600}{800}\right)}}=249 \text { qty. / production cycle }
$$

- high EPQ

$$
E P Q_{h}=\sqrt{\frac{2 K_{h} D_{h}}{h_{h}\left(1-\frac{D_{h}}{P_{h}}\right)}}=\sqrt{\frac{2 \times 180 \times 900}{16 \times\left(1-\frac{900}{1100}\right)}}=335 \text { qty. / production cycle }
$$

According to the periods of time and the impact of EPQ on profit, the following two cases are considered:

Case 1: profitable production: with low costs, high demand and production rate

$$
E P Q_{1}=\sqrt{\frac{2 K_{l} D_{m}}{h_{l}\left(1-\frac{D_{m}}{P_{h}}\right)}}=\sqrt{\frac{2 \times 100 \times 900}{7 \times\left(1-\frac{900}{1100}\right)}}=367 \text { qty./ production cycle }
$$

Case 2: unprofitable production, due to the high costs and low demand and production rate

$$
\mathrm{EPQ}_{2}=\sqrt{\frac{2 K_{h} D_{l}}{h_{h}\left(1-\frac{D_{l}}{P_{l}}\right)}}=\sqrt{\frac{2 \times 180 \times 400}{16 \times\left(1-\frac{400}{700}\right)}}=144 \text { qty./ production cycle }
$$




\section{B. Testing fuzzy ELQ}

Considering the historical evolution of costs, demand and production rate and the data from Table 2, the input variables of ELQ model are formulated and fuzzified in triangular fuzzy numbers. In the formulation process, the following assumptions are considered:

- all crisp values of input variables are grouped in three categories described by the linguistic terms: low, medium or reasonable and high;

- the linguistic terms consider the past evolution of the variables and the time when these variables occurred. There are three historical periods, identified by the decision makers: periods with financial distress (characterized through cost cutting), periods with expanding objectives (characterized through investing in new products, in new assets) and periods of stagnations.

- the triangular fuzzy numbers and the membership functions are formulated using the frequency of occurrence of each crisp value of input variables, in the above-mentioned periods.

- the membership degree is designed, also, using the frequency of occurrence in the past, and means the degree of realisation in the future. It is considered that, if a variable value occurred more times in a type of period, then if the company goes through the same period, the degree of realisation in the future is $100 \%$. If the decision makers can not forecast precisely the type of period or the variable values did not occur frequently in the past, the degree of realisation is reduced;

- the output variable is formulated considering the past impact of the EPQ values on the profit level;

- the limits and the peak of output variable, ELQ, are determined considering the traditional EPQ levels (low, medium, high).

In MATLAB, the formulation and fuzzification of the input and output variables is realised through the following graphs:

- setup cost:

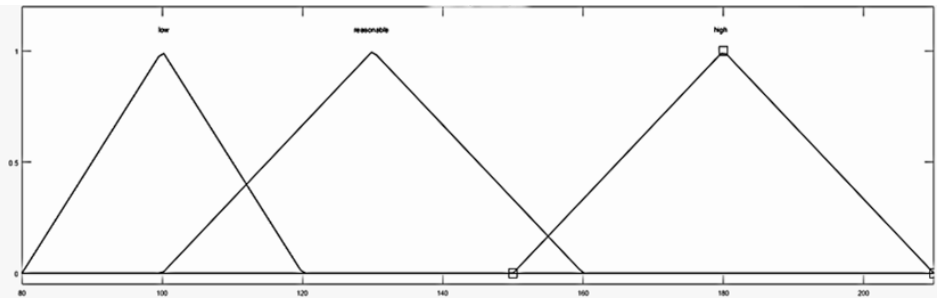

Figure Nr. 6 Fuzzy Setup Cost numbers

- demand

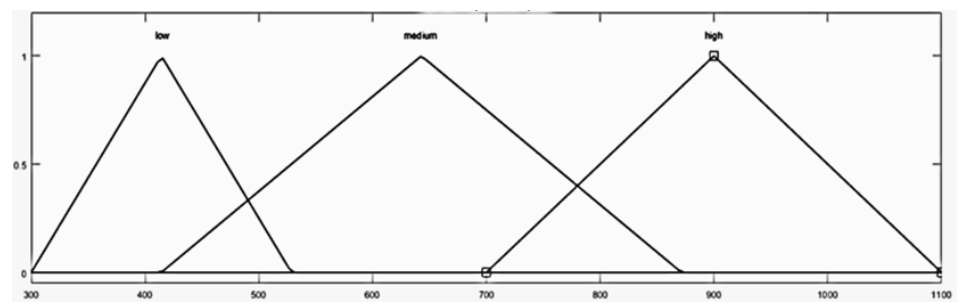

Figure Nr. 7 Fuzzy Demand numbers

- holding cost 
Figure Nr. 8 Fuzzy Holding Cost numbers

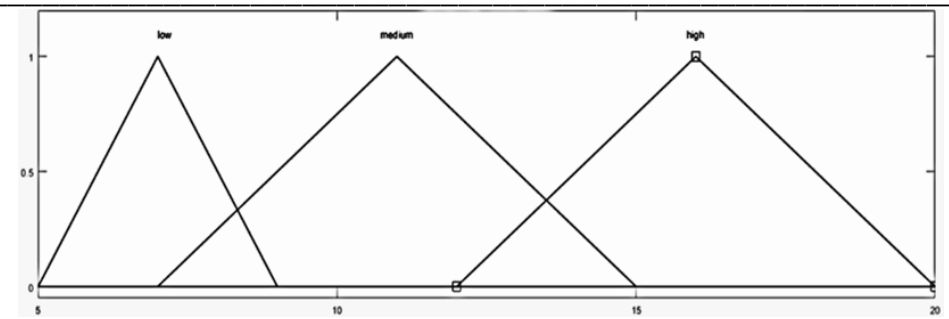

- production rate

Figure Nr. 9 Fuzzy Production Rate numbers

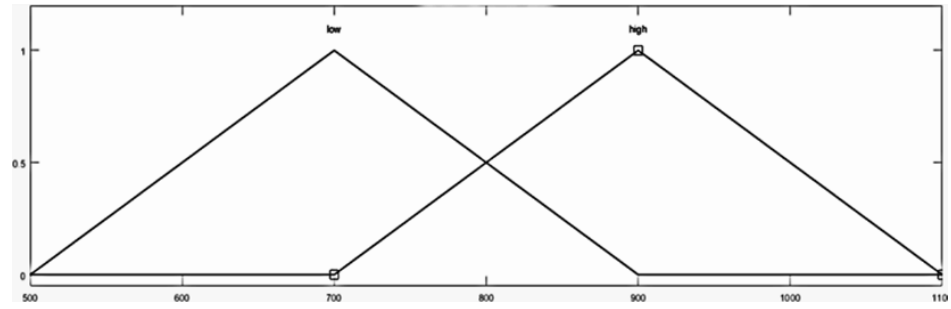

- ELQ

Figure Nr. 10 Fuzzy ELQ

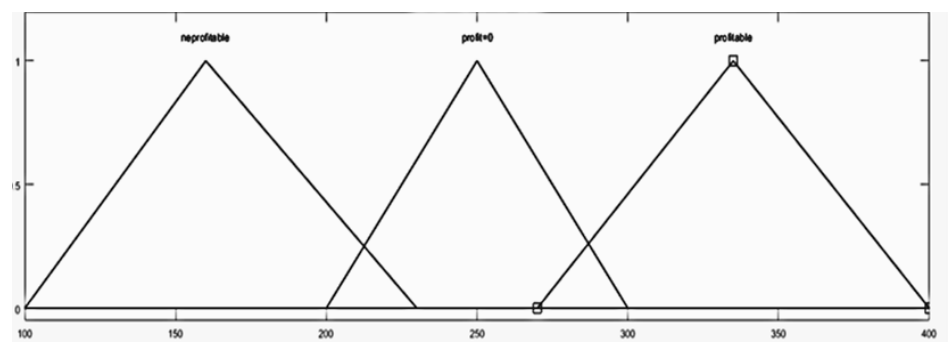

After the fuzzification, the next step of the FLS is inference, which is MAMDANI. The rule base for this inference is defined in Appendix $A$ and evaluates the impact of the input variables in the ELQ output. To evaluate the impact, the Case 1 and Case 2 are considered. In traditional EPQ model, first case provides a solution for a profitable production and it was equal to 367 qty. In the second case, the traditional EPQ is equal to 144 qty. and it is relevant for an unprofitable production.

\section{Case 1}

In the EPQ model, the following values for input variables were considered:

- Setup cost $=\$ 100$

- Demand $=900$ qty

- Holding cost $=\$ 7$

- Production rate $=1.100$ qty.

Through MAMDANI inference, the ELQ solution for these input values is equal to 335 qty, which is the high level of $E P Q$, reached in the periods with profitable production. This solution is provided by using fuzzy function in MATLAB workspace (Appendix B) and it is different from the traditional EPQ solution. The deviation between EPQ and ELQ is equal to 32 qty. The cost of the deviation is determined as follows:

$$
C_{32 q t y}=32 \times(\text { Setup Cost }+ \text { Holding Cost })=\$ 3.424
$$


This platform allows decision makers to evaluate the impact of the inputs on the ELQ, through some surfaces, which could take in consideration only two inputs from all inputs. Thus, there could be 12 surfaces that allows managers to evaluate the impact of inputs on the output. From these 12, only 4 are considered:

- the surface with the setup cost and demand inputs;

- the surface with setup cost and production rate inputs;

- the surface with the demand and production rate inputs;

- the surface with the holding cost and setup cost inputs.

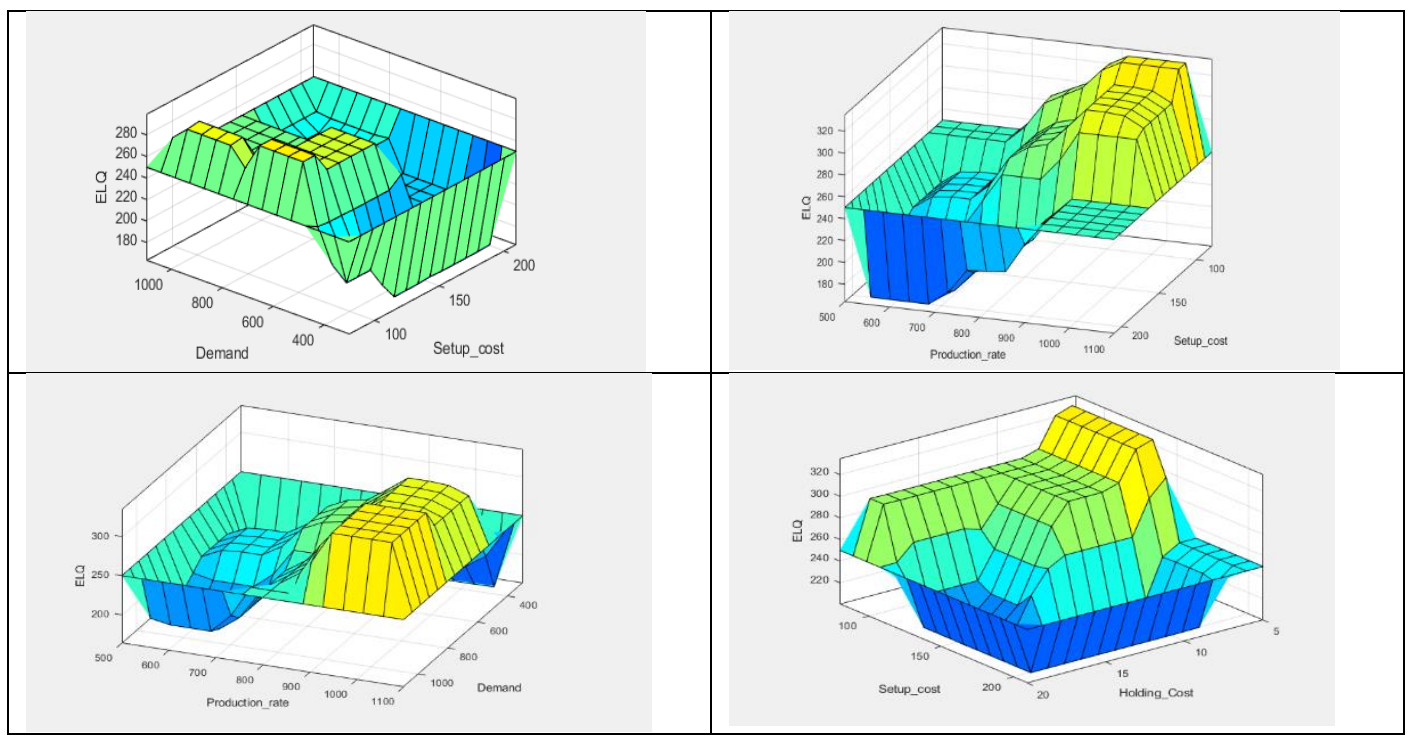

Figure Nr. 11 ELQ surfaces with low costs and high demand and production rate

Analysing, these surfaces, the decision makers could formulate the following conclusions: - if the costs are low and the demand and production rate are high, the ELQ is characteristic for a profitable production;

- if the demand and production rate are medium, the ELQ is on the zero profit level;

- the setup cost has a greater impact than holding cost on the ELQ.

\section{Case 2}

In the second EPQ traditional case, the following values of the input variables were considered:

- Setup cost $=\$ 180$

- Demand $=400$ qty

- Holding cost $=\$ 16$

- Production rate $=700$ qty.

Considering these values, the EPQ was equal to 144 qty. and was relevant for an unprofitable production, due to the high costs. Through Mamdani inference, using the rule base defined in Appendix C, the ELQ is equal to 163, with extra 19 qty., comparing to EPQ. This extra quantity has a cost determined as follows:

$$
C_{19 q t y}=19 \times(\text { Setup Cost }+ \text { Holding Cost })=\$ 54.720
$$

It can be observed that in first case, the EPQ has an extra cost and in the second case, the ELQ has an extra cost. The impact of these extra costs on the profit level, could be a future research direction and it is important direction, in order to provide a complex solution for the decision makers. 


\section{Conclusions and recommendations}

In modelling and testing ELQ, the following strengths and weaknesses can be identified:

\section{Strengths:}

- The ELQ model allows managers to formulate decision problems, even if the parameters are vague;

- The ELQ model allows managers to identify the optimal quantity to be produced and the impact of the optimal quantity on the profit level;

- The ELQ model, through MATLAB interface, allows decision makers to simulate every time when there are changes in input variables, and to obtain the solution, in the shortest time possible;

\section{Weaknesses:}

- If there are many input variables, the rule base, used in ELQ model, is very time consuming; - The rule base appeals to the decision makers experience and there can occur some errors in formulating the rules;

- The data collection process needs some algorithms to rank the variables and to formulate the membership function of these variables.

The ELQ model can be developed in a such way that the weaknesses would be eliminated and decision process would be have less errors:

- The ELQ model could consider variables as: quality, backorders, discounted price. The solution provided by this complex ELQ would be more authentic and valid;

- The ELQ, combined with Sensitivity Analysis would help decision makers to measure the impact of an extra quantity on the profit level.

- The ELQ model, combined with AHP algorithm would help managers in collecting and ranking input and output variable

- The ELQ model developed using recursive algorithms would help decision makers to formulate automatically the rule base.

\section{Acknowledgement}

The work was supported by University of Oradea, Doctoral School of Economic Science.

\section{References}

1. Viji, G., \& Karthikeyan, K. (2018). An economic production Quantity model for three levels of production WITH Weibull distribution deterioration and shortage. Ain Shams Engineering Journal, 9(4), 1481-1487. DOI:10.1016/j.asej.2016.10.006

2. Kaur, D. A., \& Kaur, K. (2009). Fuzzy expert systems based on MEMBERSHIP functions and fuzzy rules. 2009 International Conference on Artificial Intelligence and Computational Intelligence. DOI:10.1109/aici.2009.382

3. Lee, H., \& Yao, J. (1998). Economic production quantity for fuzzy demand quantity, and fuzzy production quantity. European Journal of Operational Research, 109(1), 203-211. DOI:10.1016/s03772217(97)00200-2

4. Lin, D., \& Yao, J. (2000). Fuzzy economic production for production inventory. Fuzzy Sets and Systems, 111(3), 465-495. DOI:10.1016/s0165-0114(98)00037-2, [online], Available: https://www.sciencedirect.com/science/article/abs/pii/S0165011498000372?via\%3Dihub

5. Hsieh, C. H. (2002). Optimization of fuzzy production inventory models. Information Sciences, 146(1-4), 29-40. DOI: 10.1016/s0020-0255(02)00212-8, [online], Available: https://scihub.st/10.1016/S0020-0255(02)00212-8

6. Chen, S. H., \& Chang, S. M. (2008). Optimization of fuzzy production inventory model with unrepairable defective products. International Journal of Production Economics, 113(2), 887-894. DOI:10.1016/j.ijpe.2007.11.004, [online], Available: https://sci-hub.st/10.1016/j.ijpe.2007.11.004

7. Paul, S. K., Azeem, A., Sarker, R., \& Essam, D. (2013). Development of a production inventory model with uncertainty and reliability considerations. Optimization and Engineering, 15(3), 697-720. DOI:10.1007/s11081-013-9218-6, [online], Available: https://sci-hub.st/10.1007/s11081-013-9218-6 
8. Roy, A., Maity, K., Kar, S., \& Maiti, M. (2009). A production-inventory model with remanufacturing for defective and usable items in fuzzy-environment. Computers \& Industrial Engineering, 56(1), 8796. DOI:10.1016/j.cie.2008.04.004, [online], Available: https://sci-hub.st/10.1016/j.cie.2008.04.004

9. Islam, S., \& Roy, T. K. (2006). A fuzzy EPQ model with flexibility and RELIABILITY consideration and DEMAND dependent unit production cost under a Space Constraint: A fuzzy geometric programming approach. Applied Mathematics and Computation, 176(2), 531-544. DOI:10.1016/j.amc.2005.10.001, [online], Available: https://sci-hub.st/10.1016/i.amc.2005.10.001

10. Mandal, N. K., \& Roy, T. K. (2006). A displayed inventory model with I-r fuzzy number. Fuzzy Optimization and Decision Making, 5(3), 227-243. DOl:10.1007/s10700-006-0012-1, [online], Available: https://sci-hub.st/10.1007/s10700-006-0012-1

11. Zhang, C., Zhao, R., \& Tang, W. (2009). Optimal run lengths in deteriorating production processes in random fuzzy environments. Computers \& Industrial Engineering, 57(3), 941-948. DOI:10.1016/j.cie.2009.03.014, [online], Available: https://sci-hub.st/10.1016/i.cie.2009.03.014

12. De, S. K., \& Sana, S. S. (2014). A multi-periods production-inventory model with capacity constraints for multi-manufacturers - a global optimality in intuitionistic fuzzy environment. Applied Mathematics and Computation, 242, 825-841. DOI:10.1016/j.amc.2014.06.075, [online], Available: https://sci-hub.st/10.1016/i.amc.2014.06.075

13. Chakrabortty, S., Pal, M., \& Nayak, P. K. (2013). Intuitionistic fuzzy optimization technique for Pareto optimal solution of Manufacturing INVENTORY models with shortages. European Journal of Operational Research, 228(2), 381-387. DOI:10.1016/j.ejor.2013.01.046, [online], Available: https://sci-hub.st/10.1016/..ejor.2013.01.046

14. DE, S. K., \& MAHATA, G. C. (2019). An EPQ model FOR Three-layer supply chain with PARTIAL backordering And disruption: Triangular DENSE Fuzzy lock set approach. Sādhanā, 44(8). DOI:10.1007/s12046-019-1160-7, [online],

Available: https://www.ias.ac.in/article/fulltext/sadh/044/08/0177

15. Mira, M. \& Rubaiee, Saeed \& Ahmed, Anas \& Alroobaea, Roobaea \& Ghiduk, Ahmed. (2018). Fuzzy Logic Based Model For Optimizing Inventory Cost. Interdisciplinary Journal of Information. 21, [online], Available: https://www.researchgate.net/publication/325128364 Fuzzy Logic Based Model For Optimizing I nventory Cost

16. Antão, R. (2017). Type-2 fuzzy logic. Nonlinear Physical Science. DOI:10.1007/978-981-10-46339,_[online], Available: https://2lib.org/book/3396451/170a10 
The Annals of the University of Oradea. Economic Sciences

TOM XXX, $1^{\text {st }}$ Issue, July 2021

\begin{tabular}{|c|c|c|c|c|c|}
\hline \multicolumn{6}{|l|}{ Appendix A } \\
\hline $\begin{array}{l}\text { Nr. } \\
\text { Crt. }\end{array}$ & $\begin{array}{l}\text { Setup } \\
\text { Cost }\end{array}$ & Demand & $\begin{array}{l}\text { Holding } \\
\text { Cost }\end{array}$ & $\begin{array}{l}\text { Production } \\
\text { Rate }\end{array}$ & $\begin{array}{c}\text { Economic Logic } \\
\text { Quantity (ELQ) }\end{array}$ \\
\hline 1 & low & low & low & low & unprofitable \\
\hline 2 & low & low & low & high & profit $=0$ \\
\hline 3 & low & low & reasonable & low & unprofitable \\
\hline 4 & low & low & reasonable & high & profit $=0$ \\
\hline 5 & low & low & high & low & unprofitable \\
\hline 6 & low & low & high & high & unprofitable \\
\hline 7 & low & medium & low & low & profitable \\
\hline 8 & low & medium & low & high & profitable \\
\hline 9 & low & medium & reasonable & low & profit $=0$ \\
\hline 10 & low & medium & reasonable & high & profitable \\
\hline 11 & low & medium & high & low & profit $=0$ \\
\hline 12 & low & medium & high & high & profitable \\
\hline 13 & low & high & low & low & profitable \\
\hline 14 & low & high & low & high & profitable \\
\hline 15 & low & high & reasonable & low & profitable \\
\hline 16 & low & high & reasonable & high & profitable \\
\hline 17 & low & high & high & low & unprofitable \\
\hline 18 & low & high & high & high & profit $=0$ \\
\hline 19 & reasonable & low & low & low & unprofitable \\
\hline 20 & reasonable & low & low & high & profit $=0$ \\
\hline 21 & reasonable & low & reasonable & low & unprofitable \\
\hline 22 & reasonable & low & reasonable & high & unprofitable \\
\hline 23 & reasonable & low & high & low & unprofitable \\
\hline 24 & reasonable & low & high & high & unprofitable \\
\hline 25 & reasonable & medium & low & low & profitable \\
\hline 26 & reasonable & medium & low & high & profitable \\
\hline
\end{tabular}


The Annals of the University of Oradea. Economic Sciences

TOM XXX, $1^{\text {st }}$ Issue, July 2021

\begin{tabular}{|c|c|c|c|c|c|}
\hline $\begin{array}{l}\text { Nr. } \\
\text { Crt. }\end{array}$ & $\begin{array}{l}\text { Setup } \\
\text { Cost }\end{array}$ & Demand & $\begin{array}{l}\text { Holding } \\
\text { Cost }\end{array}$ & $\begin{array}{l}\text { Production } \\
\text { Rate }\end{array}$ & $\begin{array}{c}\text { Economic Logic } \\
\text { Quantity (ELQ) }\end{array}$ \\
\hline 27 & reasonable & medium & reasonable & low & profit $=0$ \\
\hline 28 & reasonable & medium & reasonable & high & profitable \\
\hline 29 & reasonable & medium & high & low & unprofitable \\
\hline 30 & reasonable & medium & high & high & profit $=0$ \\
\hline 31 & reasonable & high & low & low & profitable \\
\hline 32 & reasonable & high & low & high & profitable \\
\hline 33 & reasonable & high & reasonable & low & unprofitable \\
\hline 34 & reasonable & high & reasonable & high & profitable \\
\hline 35 & reasonable & high & high & low & profit $=0$ \\
\hline 36 & reasonable & high & high & high & profitable \\
\hline 37 & high & low & low & low & profit $=0$ \\
\hline 38 & high & low & low & high & unprofitable \\
\hline 39 & high & low & reasonable & low & unprofitable \\
\hline 40 & high & low & reasonable & high & unprofitable \\
\hline 41 & high & low & high & low & unprofitable \\
\hline 42 & high & low & high & high & unprofitable \\
\hline 43 & high & medium & low & low & profit $=0$ \\
\hline 44 & high & medium & low & high & profit $=0$ \\
\hline 45 & high & medium & reasonable & low & unprofitable \\
\hline 46 & high & medium & reasonable & high & profit $=0$ \\
\hline 47 & high & medium & high & low & unprofitable \\
\hline 48 & high & medium & high & high & profit $=0$ \\
\hline 49 & high & high & low & low & unprofitable \\
\hline 50 & high & high & low & high & profitable \\
\hline 51 & high & high & reasonable & low & unprofitable \\
\hline 52 & high & high & reasonable & high & profit $=0$ \\
\hline 53 & high & high & high & low & unprofitable \\
\hline
\end{tabular}


The Annals of the University of Oradea. Economic Sciences TOM XXX, $1^{\text {st }}$ Issue, July 2021

\begin{tabular}{|c|c|c|c|c|c|}
\hline $\begin{array}{c}\text { Nr. } \\
\text { Crt. }\end{array}$ & $\begin{array}{c}\text { Setup } \\
\text { Cost }\end{array}$ & Demand & $\begin{array}{c}\text { Holding } \\
\text { Cost }\end{array}$ & $\begin{array}{c}\text { Production } \\
\text { Rate }\end{array}$ & $\begin{array}{c}\text { Economic Logic } \\
\text { Quantity (ELQ) }\end{array}$ \\
\hline \hline 54 & high & high & high & high & profitable \\
\hline
\end{tabular}


The Annals of the University of Oradea. Economic Sciences

TOM XXX, $1^{\text {st }}$ Issue, July 2021

\section{Appendix B}

\section{Case 1. ELQ Test}

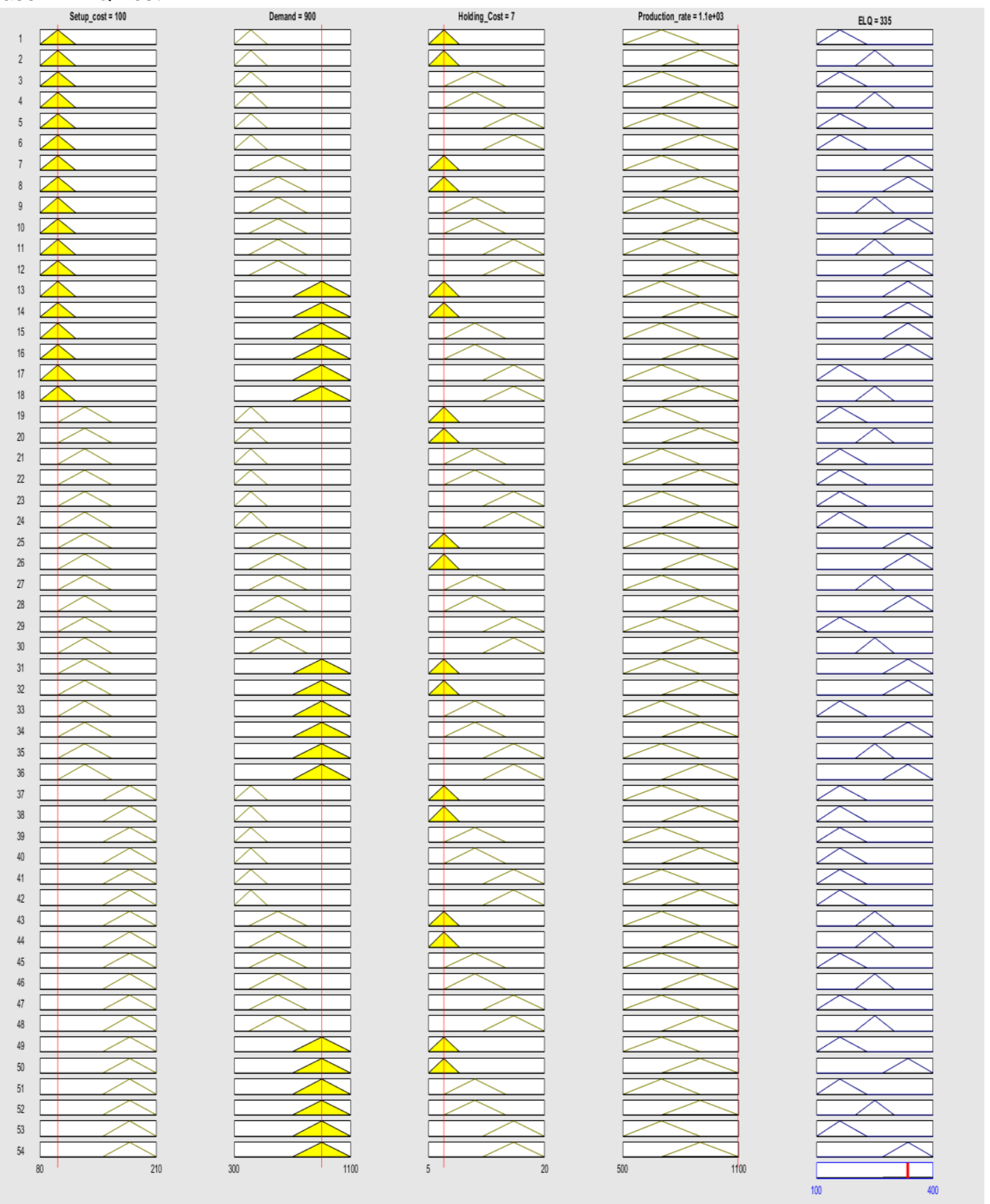


The Annals of the University of Oradea. Economic Sciences

TOM XXX, $1^{\text {st }}$ Issue, July 2021

\section{Appendix C}

Case 2. ELQ Test

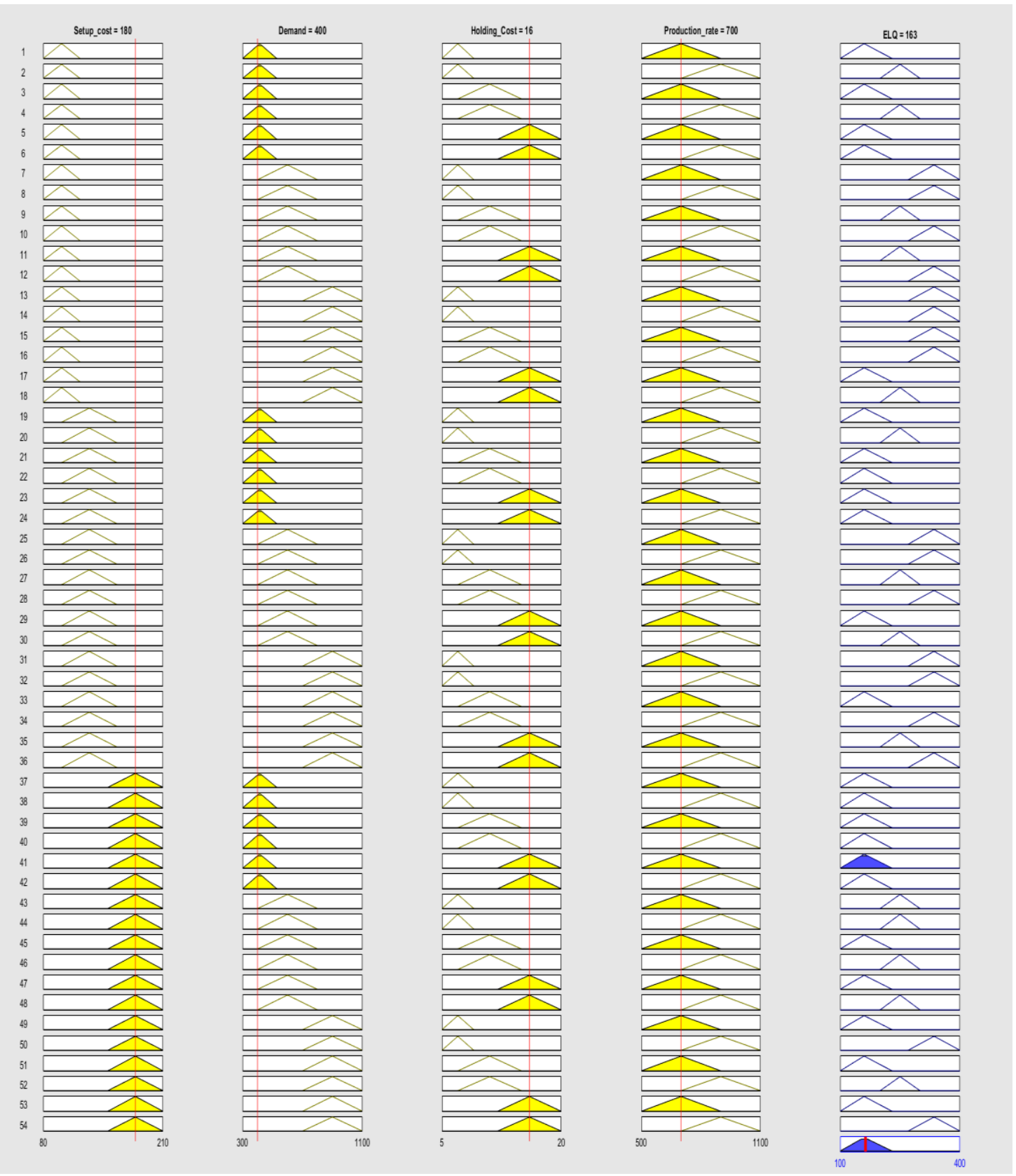

